\title{
DIGITALCOMMONS
}

$5-1-2002$

\section{Combining Two Nonparametric Tests Of Location}

R. Clifford Blair

University of South Florida

Follow this and additional works at: http://digitalcommons.wayne.edu/jmasm

Part of the Applied Statistics Commons, Social and Behavioral Sciences Commons, and the Statistical Theory Commons

\section{Recommended Citation}

Blair, R. Clifford (2002) "Combining Two Nonparametric Tests Of Location," Journal of Modern Applied Statistical Methods: Vol. 1 : Iss. 1 , Article 3.

DOI: $10.22237 /$ jmasm/ 1020254760

Available at: http://digitalcommons.wayne.edu/jmasm/vol1/iss1/3 


\title{
Combining Two Nonparametric Tests Of Location
}

\author{
R. Clifford Blair \\ Department of Epidemiology and Biostatistics \\ College of Public Health, \& \\ Jaeb Center For Health Research \\ University Of South Florida
}

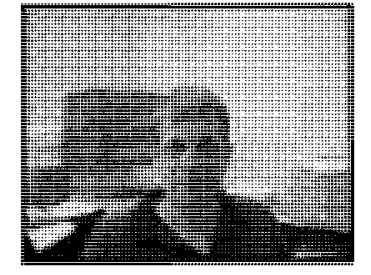

A distribution-free test is proposed whose power is similar to that of the Wilcoxon Rank-Sum or Terry-Hoeffding Normal Scores tests depending on which of these two tests is more powerful in a given data analysis situation, regardless of the population. This new statistic is distribution-free, and adds no new assumptions to those associated with the constituent tests. A table of critical values for the new statistic is given and some of its Type I error and power properties are examined.

Key words: Nonparametric tests, Shift in location, Wilcoxon rank-sum, Terry-Hoeffding, Normal scores

\section{Introduction}

Researchers are sometimes presented with situations in which two (or more) statistical tests appear to be equally appropriate for a given data analysis problem. In choosing between these tests the researcher may consider such factors as ease of computation, acceptability by peers, and availability of tables of critical values. Among the more important factors to influence such a choice would be the relative power of the statistics under consideration. Ceteris Paribus, one would desire to use the most powerful test available.

Unfortunately, it does not usually occur in such cases that one test is more powerful than its competitor among all plausible population models that may be appropriate for the data in the sample. Instead, one test or another may be more powerful than its primary competitor under a given set of circumstances. Thus, for example, one test might be preferred when the population has a lighttailed distribution, but may give way to its rival statistic when the distribution is heavy-tailed.

Factors that influence a test's power may be difficult to assess from available data. Moreover, these factors may interact in such complex patterns as to preclude any clear indication as to which test might be more powerful in a given situation. For certain inferential tests, the dilemma of test choice can be avoided through use of a "maximum" statistic (Cox, 1977). In essence, a maximum statistic is obtained by computing two or more statistics on a given data set, and choosing as the test statistic the one with the smallest associated $\mathrm{p}$-value.

R. Clifford Blair is Professor and Interim Chair, Department of Epidemiology and Biostatistics, College of Public Health, University of South Florida, Tampa, Florida. His areas of expertise are in computer-intensive statistical methods, multiple end point analysis, and control of family-wise error.
Consider that two independent samples layout. Two robust and powerful competitors are the Wilcoxon Rank-Sum test (W) and its normal scores counterpart, the Terry-Hoeffding (NS) (Terry, 1952) counterpart. Both procedures are used to test the null hypothesis that samples are from a common population. Asymptotic results suggest that these two tests may manifest substantial power differences, with the magnitudes and advantages of such differences depending on the shape of the population. The Asymptotic Relative Efficiencies (AREs) indicate that, in general, when alternatives are expressed as simple shifts in location, the normal scores test is more efficient than the rank test when sampling is from a light-tailed distribution. However, the normal scores test is at a disadvantage when the populations are heavy-tailed. (For details, see Chernoff and Savage, 1958; Hodges \& Lehmann, 1961; Lehmann, 1959; Mikulski, 1963; and Terry, 1952.)

Thus, the purpose of this paper is to present a simple maximum statistic that can be used in lieu of a choice between the Wilcoxon Rank-Sum and Terry-Hoeffding tests.

\section{Methodology}

The proposed statistic is obtained by computing both the rank-sum and the normal scores statistics, and choosing as the test statistic the one with the smaller p-value. In order to facilitate development of the sampling distribution of this maximum statistic, it is helpful to express W and NS in a common metric. In this case, both W and NS may be easily expressed in the form of a t statistic. (There are other possibilities, but existing software makes this choice computationally simpler.)

In the case of $\mathrm{W}$, this accomplished by replacing original observations with their respective ranks (with ranking being carried out without regard to group) and computing the usual independent samples $t$ statistic on those 
ranks. The resulting rank transformation statistic $\left(t_{\mathrm{w}}\right)$ is a monotone function of W (Conover \& Iman, 1981). Its sampling distribution is well approximated by a $t$ distribution with $n_{1}+n_{2}-2$ degrees of freedom (Iman, 1974).

Similarly, an expression for NS may be obtained by replacing observations with their respective normal scores, which are defined as the expected values of the order statistics under normality (Owen, 1962). The $t$ statistic is then computed on these normal scores. The resulting statistic $\left(\mathrm{t}_{\mathrm{NS}}\right)$ is a monotone function of NS, and it too may be referred to the $t$ distribution (Bradley, 1968).

Thus, the new test statistics $\left(\mathrm{t}_{\max }\right)$ is defined as

$$
\mathrm{t}_{\max }=\left\{\begin{array}{l}
+W ;|+W|>\mid+N S \\
t N S ;|+N S|>|+W|
\end{array}\right\}
$$

In the event $\left|t_{W}\right|=\left|t_{N S}\right|$, then either statistic may be used.

\section{Sampling Distribution}

The exact sampling distribution of tmax may be obtained by forming all possible permutations of the integers 1 to $n_{1}+n_{2}$ (where $n_{1}$ and $n_{2}$ represent the number of observations in each of two samples), computing $t_{\max }$ on each set of integers, and forming the cumulative distribution of the values obtained. In this study, the cumulative distribution of $\mathrm{t}_{\text {max }}$ was estimated by randomly permuting the integers $n$ to $2 n 500,000$ times with $t_{\text {max }}$ being computed on each permutation.

Table 1 provides values for $n 1=n 2=n=5(1) 40$, $40(5) 60,60(10) 120$. It should be noted that the sampling distribution of $t_{\max }$ is discrete, and therefore, it was not always possible to find critical values (c) such that $\mathrm{p}\left(\mathrm{t}_{\max } \leq\right.$ $c)=\alpha$. As a result, values of $c$ were chosen so that $\mathrm{c}$ was as large as possible, while maintaining the inequality.

It can also be seen that in some instances, the magnitude of $\mathbf{c}$ increases when $\mathbf{n}$ is increased, contrary to what is usually expected. This occurs because $t_{w}\left(t_{N S}\right)$ may be the test statistic for one particular value of $n$, and $t_{\mathrm{NS}}$ $\left(\mathrm{t}_{\mathrm{w}}\right)$ for the situation where $\mathrm{n}$ is increased. This does not lead to a violation of the above stated inequality, however, so that the test level is maintained.

\section{Results}

\section{Type I Errors}

The results of a Monte Carlo study are compiled in Table 2. The entries reflect the Type I error rates for $t_{w}$, $t_{\mathrm{NS}}$, and $\mathrm{t}_{\max }$ when samples are of various sizes. Data were generated by randomly permuting the integers from 1 to $n_{1}$ $+n_{2}$, with the three statistics being computed on each permutation. Entries in the table for $t_{w}, t_{N S}$, and $t_{\max (a)}$ were obtained by referring the three statistics to the appropriate critical values in a t table, using $n_{1}+n_{2}-2$ degrees of freedom. Entries for $t_{\max (b)}$ were obtained, in the case of $n_{1}$ $=n_{2}$, by referencing $t_{\max }$ to the critical values in Table 1. In the case of $n_{1} \neq n_{2}$, entries for $t_{\max (b)}$ were obtained by referring $\mathrm{t}_{\max }$ to the critical value in Table 1 using $\mathrm{n}=.5\left(\mathrm{n}_{1}+\right.$ $\mathrm{n}_{2}$ ). (Recall that the critical values in Table 1 were obtained under the condition of $n_{1}=n_{2}=n$.) Twenty thousand repetitions of the experiment were carried out for each condition studied.

Several points should be made regarding the results of these simulations. (1) The t distribution provides a good approximation for the distribution of $t_{w}$ and $t_{w s}$ is reaffirmed. (2) Critical values from Table 1 produce Type I error rates for $t_{\text {max }}$ near nominal levels both in the case of $n_{1}=n_{2}$ and in the case of $n_{1} \neq n_{2}$. (3) Referencing $t_{\max }$ to $a$ $t$ distribution with $n_{1}+n_{2}-2$ degrees of freedom results in only modest Type I error inflations. This result implies that the researcher who is willing to tolerate minor Type I inflations need not rely on the special table of critical values provided when conducting a test based on $t_{\text {max }}$.

\section{Power}

Let $\mathrm{P}_{t \mathrm{w}}(\alpha)$ and $\mathrm{P}_{\mathrm{tNS}}(\alpha)$ denote the power of the $\mathrm{t}_{\mathrm{w}}$ and $\mathrm{t}_{\mathrm{Ns}}$ tests, respectively, when carried out individually at the $\alpha$ level of significance. Let $\alpha^{*}>\alpha$ denote the effective level of significance of the maximum test when the critical value is chosen in this way. Because the maximum test rejects the null hypothesis when either $t_{w}$ or $t_{\text {NS }}$ is significant, it follows that the power of the maximum test a level of significance $\alpha^{*}$ has a lower bound $\max \left(\mathrm{P}_{\mathrm{nv}}(\alpha)\right.$, $\left.\mathrm{P}_{\mathrm{tNS}}(\alpha)\right)$. As indicated by the simulation above, $\alpha^{*}$ can be expected to be only slightly larger than $\alpha$. Therefore, the power of the maximum test when conducted at level of significance $\alpha$ should never be much less than the power of the better of the two individual tests when each is conducted at level of significance $\alpha$.

Figures 1-3 depict the results of a Monte Carlo study designed to compare the power of $t_{w}, t_{\mathrm{NS}}$, and $t_{\max }$. These figures indicate, respectively, the results for the normal, uniform, and Cauchy distributions. In this study, $t_{w}$ and $\mathrm{t}_{\mathrm{NS}}$ were referred to the appropriate $t$ distribution, while $t_{\max }$ was referred to the values found in Table 1 . Tests were conducted at the $\alpha=.05$ (two-tailed) level of significance. In the cases of the normal and uniform distributions, the alternative condition was constructed by adding a constant equal to $.5 \sigma$ to the scores in one group. In the case of the Cauchy distribution, which has infinite standard deviation, an arbitrary constant of 1.00 was used. Ten thousand repetitions of the experiment were carried out for each condition studied.

Figure 1 shows that there was generally little difference in the power of the three tests when sampling was from a normal population. Differences that did occur favored $t_{\mathrm{NS}}$ and $\mathrm{t}_{\max }$. In the case of the uniform distribution, Figure 2 shows that $t_{\mathrm{Ns}}$ was the most powerful test, with $t_{\max }$ showing power similar to, but slightly less than, that of 
Table 1. Two-tailed Critical Values For $t_{\max }$.

\begin{tabular}{|c|c|c|c|c|c|c|}
\hline \multirow[b]{2}{*}{$\mathrm{n}$} & \multicolumn{4}{|c|}{ Level of Significance } & \multirow[b]{2}{*}{.020} & \multirow[b]{2}{*}{.010} \\
\hline & .400 & .200 & .100 & .050 & & \\
\hline 5 & .9727 & 1.4572 & 1.8312 & 2.4958 & 3.7812 & 5.0000 \\
\hline 6 & .9571 & 1.3978 & 1.8503 & 2.2804 & 3.3211 & 3.8468 \\
\hline 7 & .9525 & 1.3919 & 1.8688 & 2.2926 & 2.7464 & 3.6623 \\
\hline 8 & .9431 & 1.4124 & 1.8066 & 2.2630 & 2.7940 & 3.4503 \\
\hline 9 & .9238 & 1.4076 & 1.8122 & 2.2005 & 2.6706 & 3.0822 \\
\hline 10 & .9027 & 1.3942 & 1.8023 & 2.1603 & 2.6616 & 2.9771 \\
\hline 11 & .9232 & 1.3744 & 1.7861 & 2.1732 & 2.6429 & 2.9582 \\
\hline 12 & .9207 & 1.3830 & 1.7872 & 2.1517 & 2.5778 & 2.9292 \\
\hline 13 & .9180 & 1.3836 & 1.7925 & 2.1339 & 2.5852 & 2.8869 \\
\hline 14 & .9162 & 1.3744 & 1.7655 & 2.1349 & 2.5764 & 2.8852 \\
\hline 15 & .9210 & 1.3682 & 1.7688 & 2.1310 & 2.5580 & 2.8463 \\
\hline 16 & .9161 & 1.3762 & 1.7613 & 2.1052 & 2.5327 & 2.8400 \\
\hline 17 & .9104 & 1.3790 & 1.7582 & 2.1188 & 2.5340 & 2.8243 \\
\hline 18 & .9154 & 1.3778 & 1.7589 & 2.1025 & 2.5128 & 2.8039 \\
\hline 19 & .9177 & 1.3737 & 1.7533 & 2.0954 & 2.5135 & 2.8152 \\
\hline 20 & .9178 & 1.3675 & 1.7486 & 2.0909 & 2.5080 & 2.8043 \\
\hline 21 & .9164 & 1.3628 & 1.7426 & 2.0826 & 2.4981 & 2.7824 \\
\hline 22 & .9137 & 1.3755 & 1.7544 & 2.0942 & 2.4956 & 2.7761 \\
\hline 23 & .9168 & 1.3640 & 1.7497 & 2.0835 & 2.4950 & 2.7876 \\
\hline 24 & .9205 & 1.3729 & 1.7485 & 2.0911 & 2.5003 & 2.7775 \\
\hline 25 & .9202 & 1.3654 & 1.7506 & 2.0763 & 2.4780 & 2.7598 \\
\hline 26 & .9142 & 1.3657 & 1.7402 & 2.0776 & 2.4769 & 2.7582 \\
\hline 27 & .9219 & 1.3692 & 1.7479 & 2.0776 & 2.4821 & 2.7618 \\
\hline 28 & .9163 & 1.3709 & 1.7476 & 2.0790 & 2.4823 & 2.7575 \\
\hline 29 & .9220 & 1.3712 & 1.7492 & 2.0817 & 2.4730 & 2.7401 \\
\hline 30 & .9200 & 1.3702 & 1.7440 & 2.0709 & 2.4652 & 2.7421 \\
\hline 31 & .9210 & 1.3683 & 1.7387 & 2.0743 & 2.4694 & 2.7491 \\
\hline 32 & .9237 & 1.3654 & 1.7441 & 2.0742 & 2.4606 & 2.7357 \\
\hline 33 & .9189 & 1.3621 & 1.7385 & 2.0703 & 2.4573 & 2.7361 \\
\hline 34 & .9189 & 1.3633 & 1.7399 & 2.0710 & 2.4545 & 2.7302 \\
\hline 35 & .9211 & 1.3665 & 1.7419 & 2.0707 & 2.4645 & 2.7340 \\
\hline 36 & .9225 & 1.3628 & 1.7364 & 2.0697 & 2.4594 & 2.7300 \\
\hline 37 & .9183 & 1.3648 & 1.7370 & 2.0693 & 2.4532 & 2.7288 \\
\hline 38 & .9237 & 1.3688 & 1.3730 & 2.0691 & 2.4561 & 2.7286 \\
\hline 39 & .9203 & 1.3623 & 1.7361 & 2.0654 & 2.4495 & 2.7129 \\
\hline 40 & .9229 & 1.3642 & 1.7345 & 2.0619 & 2.4419 & 2.7104 \\
\hline 45 & .9221 & 1.3656 & 1.7341 & 2.0664 & 2.4419 & 2.7073 \\
\hline 50 & .9221 & 1.3640 & 1.7339 & 2.0588 & 2.4444 & 2.7018 \\
\hline 55 & .9170 & 1.3595 & 1.7309 & 2.0532 & 2.4338 & 2.7012 \\
\hline 60 & .9216 & 1.3643 & 1.7320 & 2.0587 & 2.4378 & 2.7061 \\
\hline 70 & .9205 & 1.3653 & 1.7293 & 2.0487 & 2.4245 & 2.6837 \\
\hline 80 & .9210 & 1.3619 & 1.7289 & 2.0473 & 2.4205 & 2.6747 \\
\hline 90 & .9237 & 1.3635 & 1.7280 & 2.0494 & 2.4269 & 2.6840 \\
\hline 100 & .9212 & 1.3627 & 1.7288 & 2.0492 & 2.4212 & 2.6847 \\
\hline 110 & .9195 & 1.3605 & 1.7257 & 2.0419 & 2.4136 & 2.6653 \\
\hline 120 & .9205 & 1.3602 & 1.7247 & 2.0421 & 2.4150 & 2.6691 \\
\hline
\end{tabular}


Table 2. Type I Error Rates Of $t_{w}, t_{v s}$, And $t_{\text {max }}$ For Various Sample Sizes.

\begin{tabular}{|c|c|c|c|c|}
\hline \multirow[b]{2}{*}{$\mathrm{n} 1, \mathrm{n} 2$} & \multirow[b]{2}{*}{ Statistics } & \multicolumn{3}{|c|}{ Level Of Significance } \\
\hline & & .100 & .050 & $\overline{.010}$ \\
\hline \multirow[t]{4}{*}{6,6} & $t_{w}$ & .095 & .067 & .015 \\
\hline & $t_{\mathrm{ws}}^{\mathrm{w}}$ & .107 & .058 & .015 \\
\hline & $t_{\text {max }}^{\text {Ns }}$ & .111 & .067 & .015 \\
\hline & $t_{\max (b)}$ & .097 & .049 & .008 \\
\hline \multirow[t]{4}{*}{$3,9 \mathrm{t}_{\mathrm{w}}$} & $t_{w}$ & .099 & .064 & .010 \\
\hline & $t_{\mathrm{NS}}^{w}$ & .099 & .054 & .010 \\
\hline & $t_{\max (a)}$ & .109 & .064 & .010 \\
\hline & $\begin{array}{l}t_{\max (a)}(b) \\
\end{array}$ & .109 & .064 & .010 \\
\hline \multirow{4}{*}{$10,10 \mathrm{t}_{\mathrm{w}}$} & $t_{w}$ & .105 & .052 & .012 \\
\hline & $\mathrm{t}_{\mathrm{Ns}}^{w}$ & .099 & .050 & .012 \\
\hline & $t_{\max (a)}^{\text {Ns }}$ & .114 & .057 & .013 \\
\hline & $t_{\max (b)}$ & .099 & .049 & .011 \\
\hline \multirow[t]{4}{*}{$5,15 \mathrm{t}_{\mathrm{w}}$} & $t_{w}$ & .097 & .051 & .011 \\
\hline & $t_{\mathrm{NS}}$ & .100 & .050 & .011 \\
\hline & $t_{\max (a)}$ & .112 & .058 & .013 \\
\hline & $t_{\max (b)}$ & .106 & .050 & .010 \\
\hline \multirow[t]{4}{*}{20,20} & $t_{w}$ & .104 & .050 & .010 \\
\hline & $t_{\mathrm{ws}}^{w}$ & .102 & .052 & .011 \\
\hline & $t_{\max (a)}^{N s}$ & .117 & .060 & .013 \\
\hline & $\begin{array}{l}\max (a) \\
t_{\max (b)}\end{array}$ & .102 & .052 & .010 \\
\hline \multirow[t]{4}{*}{10,30} & $t_{w}$ & .101 & .047 & .010 \\
\hline & $t_{\mathrm{Ns}}^{w}$ & .098 & .047 & .009 \\
\hline & $t^{\text {NS }}$ & .116 & .055 & .011 \\
\hline & 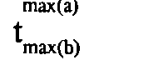 & .101 & .049 & .010 \\
\hline \multirow[t]{4}{*}{40,40} & $t_{w}$ & .100 & .049 & .010 \\
\hline & $t_{\mathrm{ss}}$ & .101 & .049 & .010 \\
\hline & $t_{\max (a)}$ & .117 & .058 & .012 \\
\hline & $\begin{array}{l}t_{\max (a)} \\
t_{\max (b)}\end{array}$ & .100 & .050 & .010 \\
\hline \multirow[t]{4}{*}{20,60} & $t_{w}$ & .101 & .052 & .009 \\
\hline & $t_{\mathrm{NS}}^{w}$ & .101 & .051 & .010 \\
\hline & $t_{\max (a)}$ & .116 & .061 & .012 \\
\hline & $t_{\max (b)}$ & .101 & .052 & .009 \\
\hline \multirow[t]{4}{*}{60,60} & $\mathrm{t}_{\mathrm{w}}$ & .101 & .049 & .010 \\
\hline & $t_{\mathrm{Ns}}^{w}$ & .101 & .048 & .010 \\
\hline & $\mathrm{t}_{\operatorname{max(a)}}$ & .117 & .058 & .012 \\
\hline & $\begin{array}{l}t_{\max (a)} \\
\max (b)\end{array}$ & .102 & .048 & .010 \\
\hline \multirow[t]{4}{*}{30,90} & $t_{w}$ & .100 & .049 & .011 \\
\hline & $t_{\mathrm{Ns}}^{w}$ & .100 & .050 & .011 \\
\hline & $\mathrm{t}_{\max (\mathrm{a})}$ & .116 & .058 & .011 \\
\hline & $\mathrm{t}_{\max (\mathrm{max})}$ & .100 & .049 & .011 \\
\hline
\end{tabular}



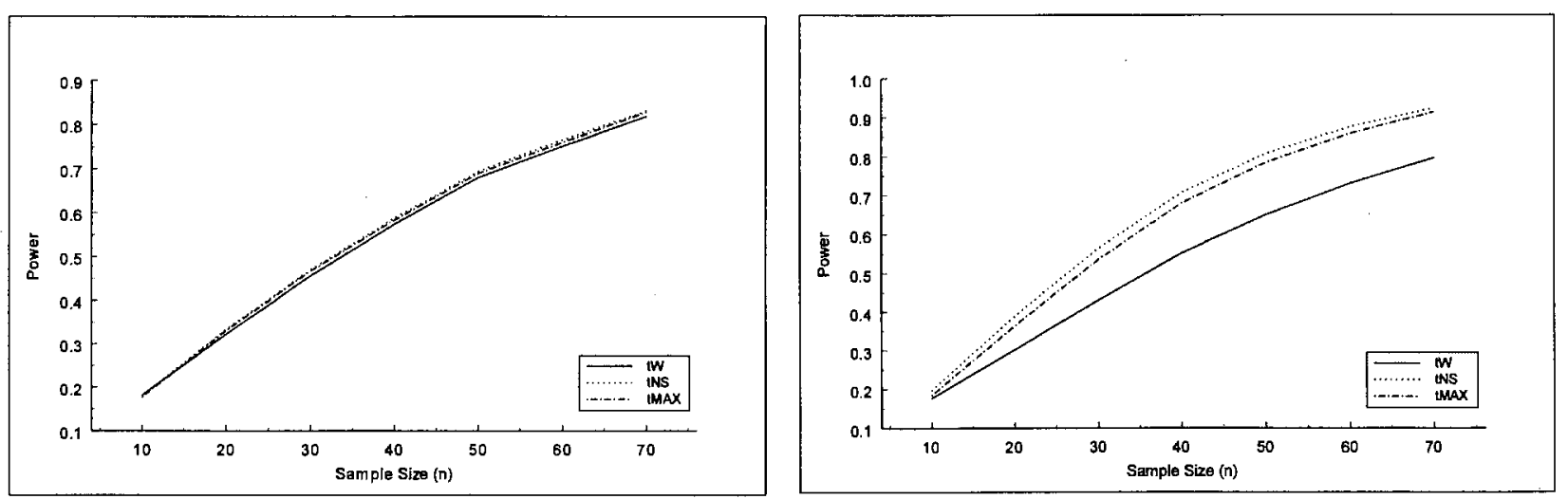

Figure 3. Power of tW, tMS and tMAX When Sampling is From a Cauchy Distriubtuon

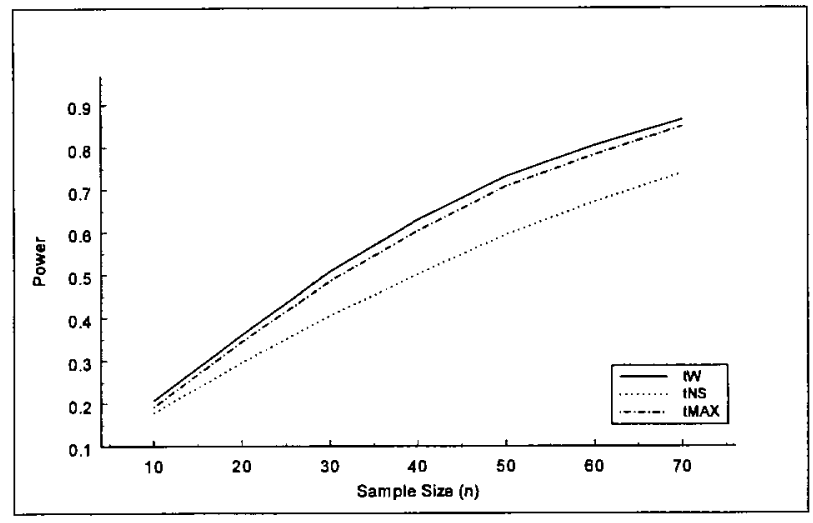

$\mathrm{t}_{\mathrm{vs}}$. Under the heavy-tailed Cauchy distribution, $\mathrm{t}_{\mathrm{w}}$ was the most powerful statistic, with $t_{\text {max }}$ once again demonstrating power similar to, but slightly less than, that of the most powerful test.

\section{Conclusion}

It is usually difficult for researchers to obtain sufficient information from a given data set so as to make reasonable choices between suitable statistical tests. It is important, therefore, that tests have power to detect broad classes of alternatives with high probability. The technique demonstrated here is a simple method for constructing such tests.

A major advantage of the test presented here lies in the fact that this test is automatically adaptive to the weight in the tail of the population from which the data were sampled. This is contrasted with various adaptive estimation procedures which require a preliminary estimate of tail weight.

It should also be noted that the maximum method may be extended to a wide variety of testing situations. For example, more than two statistics my be formed into a maximum test, with component tests being both parametric and non-parametric. A large number of other possibilities exist.

\section{References}

Bradley, J. V. (1968). Distribution-free statistical tests. Englewood Cliffs, NJ: Prentice-Hall.

Chernoff, H., \& Savage, I. R. (1958). Asymptotic normality and efficiency of certain nonparametric test statistics. Annals of Mathematical Statistics, 28, 972-994.

Conover, W. J., \& Iman, R. (1981). Rank transformations as a bridge between parametric and nonpara- 
metric statistics. The American Statistician, 35, 124-129. Cox, D. R. (1977). The role of significance tests. Scandinavian Journal of Statistics, 4, 49-70.

Hodges, J. L., \& Lehmann, E. L. (1961). Comparison of the normal scores and Wilcoxon tests. In (J. Neyman, ed.) Proceedings of the Fourth Berkeley Symposium on Mathematical Statistics and Probability, 1. Berkeley and Los Angeles, CA: University of California Press. Iman, R. L. (1974). An approximation to the exact distribution of the Wilcoxon-Mann-Whitney rank sum test statistic. Communications in Statistics, 5, 587-598.

Lehmann, E. L. (1959). Testing statistical hypotheses. NY: Wiley.

Mikulski, P. W. (1963). On the efficiency of optimal nonparametric procedures in the two sample case. Annals of Mathematical Statistics, 34, 22-32.

Owen, D. B. (1962). Handbook of statistical tables. Reading, MA: Addison-Wesley.

Terry, M. E. (1952). Some rank order tests which are most powerful against specific parametric alternatives. Annals of Mathematical Statistics, 23, 346-366. 